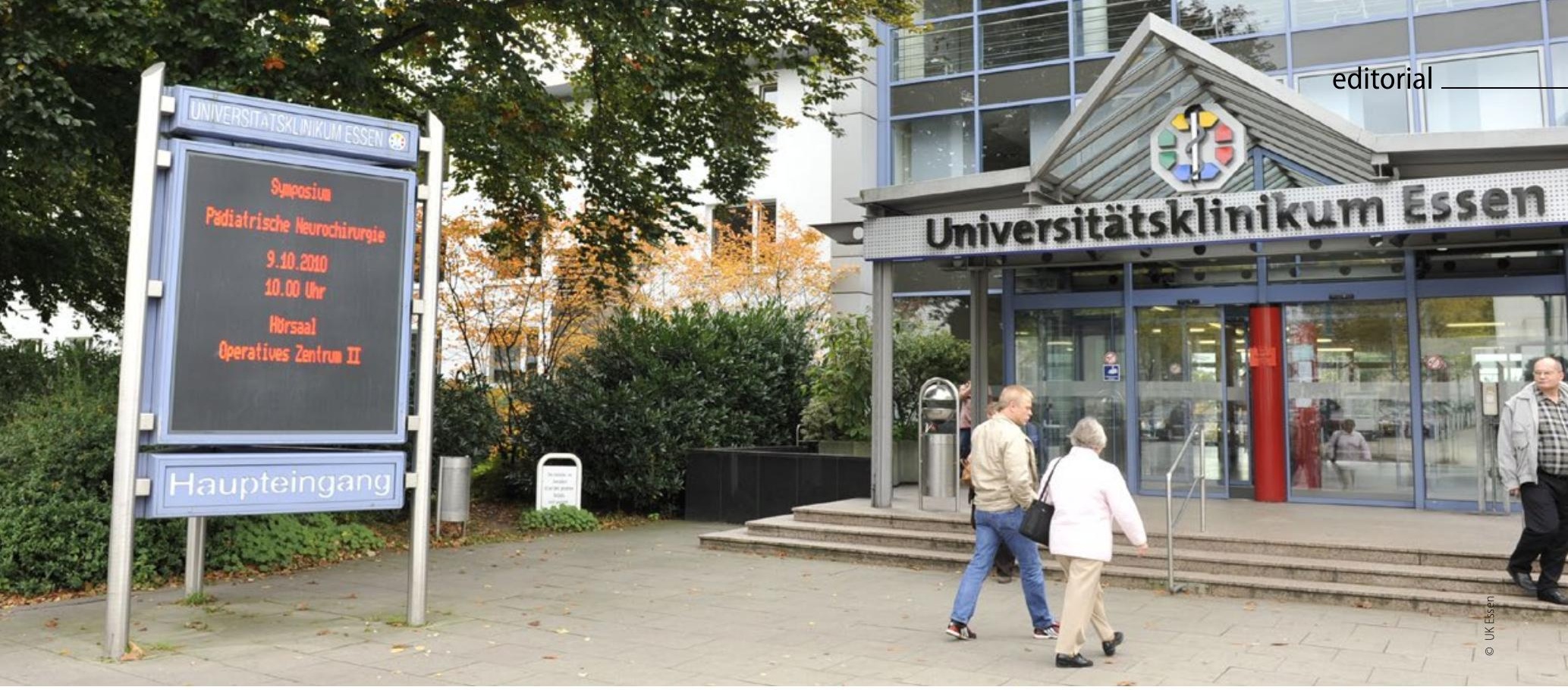

\title{
MDK - ein Risikofaktor für Universitätskliniken?
}

Der Medizinische Dienst der Krankenversicherungen, kurz MDK, wurde ursprünglich einmal als unabhängige Struktur im Gesundheitswesen installiert, um beispielsweise die folgenden medizinischen und sozialmedizinischen Fragen zu beurteilen:

- Grad der persönlichen Pflegebedürftigkeit

- Qualität einer Pflegeeinrichtung oder eines Pflegedienstes

- Beurteilung anstehender Vorsorge- und Rehabilitationsmaßnahmen

- Verdacht auf Behandlungsfehler

- Neue Untersuchungs- oder Behandlungsmethoden

- Unklarheiten bei Krankenhausrechnungen

Für den Bereich der Krankenhäuser war es sicher initial sinnvoll, in dem komplexen System des DRG-Systems und des Operationen- und Prozedurenschlüssels (OPS) Krankenhausrechnungen stichprobenartig zu überprüfen, da es in der Anfangsphase nach der Umstellung des Vergütungssystems immer wieder zu einzelnen Fehlabrechnungen kam. Anfangs erfolgten die Prüfungen von Krankenhausrechnungen tatsächlich auch stichprobenweise. In der Zwischenzeit hat sich die Überprüfung der Krankenhausrechnungen zu einem ganz eigenen Geschäftsfeld des MDK entwickelt. An einzelnen Universitätskliniken werden bis zu $50 \%$ aller Fälle und Abrechnungen überprüft. Man gewinnt zunehmend den Eindruck, dass es nicht mehr primär darum geht, nicht sachgemäße Abrechnungen zu finden, sondern dass es Ziel der Überprüfung ist, Kosten für die Krankenkassen einzusparen. So gibt es viele neurologische Universitätskliniken, die im ersten Quartal 2016 Kürzungen der Vergütungen für stationäre Patienten durch den MDK um bis zu $100.000 €$ hinnehmen mussten. Auch wenn einige dieser Kürzungen sachlich gerechtfertigt sein mögen, gibt es doch viele Beispiele, die vermuten lassen, dass es mehr um Einsparungen als um Verbesserung der Qualität geht. Ich will im Folgenden einige Beispiele nennen, die sich bei uns zugetragen haben oder die mir

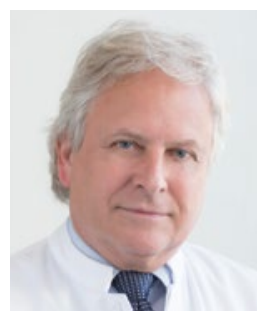

Prof. Dr. med. Hans-Christoph Diener, Essen

Seniorprofessor Klinische Neurowissenschaften Universität Duisburg-Essen E-Mail: hans.diener@uk-essen.de

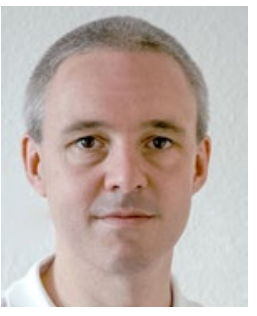

Dr. med. Peter Lütkes, Essen

Leiter Medizinisches Controlling

Universitätsklinikum Essen

E-Mail: peter.luetkes@uk-essen.de

von leitenden Ärzten anderer neurologischer Kliniken genannt wurden:

- Ein Patient wird nach einem ischämischen Insult vier Tage auf der Stroke Unit behandelt. In der Krankenakte fehlt ein einziger Blutdruckwert, der in der OPS-Komplexbehandlung des Schlaganfalls vorgesehenen Blutdruckkontrollen. Die OPS „Neurologische Komplexbehandlung des akuten Schlaganfalls" (Amtsdeutsch für: Behandlung auf der Stroke Unit) wurde gestrichen.

- Ein Patient befindet sich nach einem ischämischen Insult auf der Stroke Unit. Am dritten Tag wird um 15.00 Uhr die Krankengymnastin erwartet. Der Patient wird zum selben Zeit- 
punkt zur Kernspintomografie abgerufen. Als er auf die Station zurückkommt, ist die Dienstzeit der Physiotherapie beendet. Der MDK bemängelt, dass an diesem Tag keine Krankengymnastik stattgefunden hat und streicht die OPS.

- Ein Patient wird nach einem Schlaganfall von der Stroke Unit auf die Allgemeinstation verlegt. Er ist schwer betroffen, nach Meinung der behandelnden Ärzte aber rehabilitationsfähig. Der ursprüngliche Antrag auf Verlegung in eine Rehabilitationsklinik wird von der Krankenkasse abgelehnt. Es wird ein erneuter, deutlich detaillierterer Antrag gestellt, der nach vier Tagen positiv beschieden wird. Die Rechnung wird jetzt wegen Überschreitung der oberen Grenzverweildauer gekürzt.

- Ein Patient kommt mit einem schweren Halbseitensyndrom und die CT-Angiografie zeigt einen distalen Verschluss der A. carotis interna. Schon in der Computertomografie beginnt die systemische Thrombolyse mit rt-PA und der Patient wird anschließend in der Angiografie erfolgreich thrombektomiert. Der MDK verweigert die Bezahlung der Thrombolyse, da der Patient ja durch die Thrombektomie „geheilt“ worden sei.

- Ein Patient ist wegen Vorhofflimmern antikoaguliert, erleidet einen ischämischen Insult mit Verschluss der A. cerebri media und hat bei der Aufnahme eine INR von 2,0. Es wird unter diesen Umständen auf eine systemische Thrombolyse verzichtet und der Patient thrombektomiert. Die Erstattung wird verweigert, da der MDK argumentiert, dass in den großen Studien $90 \%$ der Patienten zunächst lysiert sowie anschließend thrombektomiert wurden und wir in diesem Fall von diesem Procedere abgewichen seien.

Leider kann diese Kette von Beispielen vielfältig fortgesetzt werden. Es kristallisieren sich mehrere Ansatzpunkte in der MDKArgumentation heraus, die bedauerlicherweise auch durch die (höchst-) richterliche Rechtsprechung unterstützt werden.

Der erste Ansatzpunkt ist die gängige Praxis der buchstabengetreuen Auslegung der Anforderungen des OPS. Hier wird übersehen, dass die Mindestmerkmale im OPS „Neurologische Komplexbehandlung des akuten Schlaganfalls“ (und den anderen OPS-Kodes) Soll-Vorstellungen sind, die in der Praxis nicht zu $100 \%$ eingehalten werden können. Das ist ungefähr so, als wenn man ein MDK-Gutachten trotz sachlicher Richtigkeit nicht anerkennen würde, da es auf zwei Seiten geschliffener Argumentation einen Kommafehler enthält. Allerdings ist es leider auch so, dass die buchstabengetreue Auslegung immerhin noch eine Orientierungsmöglichkeit für die Krankenhäuser beinhaltet und einem bei $100 \%$ Erfüllung der Mindestmerkmale die Vergütung ermöglicht.

Über diese ohnehin schon hohe Hürde geht der nächste Ansatz der Kostenträger und des MDK nochmals hinaus, nämlich die Auslegung des sozialgesetzlichen Anspruches der Versicherten auf eine „ausreichende, zweckmäßige und wirtschaftliche“ Medizin (siehe SGB V u. a. $\$ \$ 2,12$ und 70). Dass eine Behandlung in einer Stroke Unit sowohl zweckmäßig als auch wirt- schaftlich ist, zweifelt noch nicht einmal der MDK an. Aber was heißt genau „ausreichend“? Hier finden sich jetzt neue Auslegungen dieses Begriffes, die ins Mark des Selbstverständnisses einer qualitativ hochwertigen und innovativen Schlaganfallversorgung treffen. Es wird nämlich nicht nur die „nicht ausreichende" (in Schulnoten: mangelhafte und ungenügende) Therapie gestrichen, sondern auch die "gute“ und „sehr gute“, also die mehr als ausreichende Behandlung - frei nach dem Motto: „Luxusmedizin müssen wir nicht bezahlen!" Beispiele hierfür sind die Gabe von Apherese-Thrombozytenkonzentraten („Pool-TK wären ausreichend gewesen“), verlängerte Aufenthalte auf der Stroke wegen intensiver Physiotherapie etc. ...

Auch wenn man es nicht glauben mag, diese Einstellung lässt sich noch weiter ins Extreme steigern: Eine Steilvorlage hat der als äußerst krankenhausfeindlich bekannte 1. Senat des BSG geliefert, der in seinen jüngsten Urteilen die neue Rechtsauslegung des „fiktiven wirtschaftlichen Alternativverhaltens“ erfunden hat. „Erfunden“ deshalb, weil diese Auslegung in keinem Gesetz, keinem Paragrafen und keiner Gesetzesbegründung zu finden ist. Gemeint ist, dass das BSG davon ausgeht, dass ein Krankenhaus durchgängig und immer verpflichtet ist, in der Rechnungslegung nicht die konkrete DRG zugrunde zu legen, sondern die (für die Krankenkasse) billigste mögliche Variante. Es reicht, dass das fiktiv möglich ist, um die Rechnungskürzung zu begründen. So könnte eine Krankenkasse argumentieren, dass eine schnellere Verlegung in die Reha fiktiv möglich gewesen wäre, wenn der Patient intensiver behandelt worden wäre. Tatsächliche Hinderungsgründe wie „kein Rehaplatz verfügbar“ oder „Physiotherapie war ausgelastet“ würden damit als unerheblich vom Tisch gewischt werden können. Wie das in der Praxis aussehen soll oder dass man mit einer konsequenten Anwendung dieser Auffassung ganze Versorgungsstrukturen rasieren würde, interessiert offenkundig weder Richter noch Kostenträger.

Die Universitätskliniken befinden sich bereits in einem erheblichen Zielkonflikt zwischen Erbringung von medizinischen Leistungen auf höchstem Niveau - insbesondere bei seltenen und komplizierten Erkrankungen - und der Vergütung durch das DRG-System. Die meisten Universitätskliniken schreiben rote Zahlen.

Wenn nun die schon nicht ausreichenden Einnahmen der Universitätskliniken durch MDK-Beurteilungen weiter massiv gekürzt werden, steht die universitäre Medizin vor der Existenzfrage. Um es nochmal klar auszudrücken: Wir sprechen uns nicht dagegen aus, dass unangemessene Leistungen oder nicht erbrachte Leistungen kontrolliert und geprüft werden. Es geht darum, den systematischen Versuch, die Leistungserstattung zu minimieren, zu verhindern. Hier wäre sicher eine gute Strategie, wenn die Führungsspitze des MDK mit den Universitätskliniken ins Gespräch käme.

Hans-Christoph Diener und Peter Lütkes, Universitätsklinikum Essen 